\title{
Well-bounded operators on general Banach spaces
}

\author{
QIngPing Cheng
}

The theory of well-bounded operators has found many applications and has formed deep connections with other areas of mathematics. For example, it has been applied successfully to Sturm-Liouville theory, Fourier analysis and multiplier theory (see [1]). Although the theory of well-bounded operators is well established, there are a number of unresolved and interesting questions which are potentially fruitful areas for further research, and there are also a few errors in the literature. The general aim of this work is to answer some of these questions, to correct and clarify certain aspects of the theory, and to establish a more complete well-bounded operator theory, including a dual theory on general Banach spaces and a theory of compact well-bounded operators.

We first give a general method to construct well-bounded operators with special properties, such as non-uniquely decomposable, non-decomposable in $X$ and non type $(B)$. This technique can be used to produce rich examples of well-bounded operators.

An operator is well-bounded if and only if its adjoint is. On the other hand, if the underlying Banach space is nonreflexive, many of the porperties that a wellbounded operator may possess do not pass to the adjoint operator. One would hope that a suitable family of projections for the adjoint of a well-bounded operator could be formed by taking the adjoints of decomposition of the identity associated with this operator. We show that on a wide range of nonreflexive Banach spaces it is not possible to do this. We give a necessary and sufficient condition under which the hoped for relationship between corresponding decompositions of the identity holds. We have also included some results on quotients and restrictions of well-bounded operators. These results also appear in [4].

In [9], Ringrose showed that if $T$ is a well-bounded operator, there exists a decomposition of the identity for $T$ whose projections commute with $T^{*}$. He conjectured that it is possible for a well-bounded operator to have a decomposition of the identity whose projections do not all commute with $T^{*}$. Turner [10] introduced scalar-type decomposable operators of class $\Gamma$ and showed that this class of operators includes all adjoints

Received 12th May, 1999

Thesis submitted to Murdoch University, September 1998. Degree approved, April 1999. Supervisors: Associate Professor K. Harrison and Dr. I Doust.

Copyright Clearance Centre, Inc. Serial-fee code: 0004-9727/99 $\$ A 2.00+0.00$. 
of well-bounded operators [10, Theorem 3.5]. Furthermore, he proved that for a wellbounded operator $T$ on a Banach space $X$, every decomposition of the identity for $T$ is an $s$-decomposition of the identity of class $X$ for $T^{*}$ whose projections commute with $T^{*}$ (see [10, Theorems 3.5 and 3.6]). This gives a negative answer to Ringrose's conjecture. That is, if $T$ is well-bounded then $T^{*}$ comutes with any decomposition of the identity for $T$.

It seems that some details of the proof of [10, Theorem 3.5] appear to have been skipped over a little lightly. We give complete proof to [10, Theorem 3.5] which will bring the solution to Ringrose's conjecture to completion. We also give a sufficient condition for a scalar-type decomposable operator of class $\Gamma$ to be a well-bounded operator decomposable in $X$.

In an attempt to overcome some of the problems surrounding general well-bounded operators, several subclasses were introduced in [9] and [2]. Two of them are a class of well-bounded operators which are decomposable in $X$ and the class of well-bounded operators of type $(A)$. The two classes have been studied extensively. It is a simple consequence of the definition that a well-bounded operator of type $(A)$ is decomposable in $X$. Several examples of well-bounded operators which are decomposible in $X$, but which are not of type $(A)$ have been given in the literature. We show, however, that none of these operators is decomposable in $X$. Furthermore, contrary to examples in the literature, we show that every well-bounded operator which is decomposable in $X$ is automatically of type $(A)$. This result corrects a mistake in the literature which has gone unnoticed for over 25 years. Some intresting consequences are given.

It is well-known that every well-bounded operator on a reflexive Banach space is of type $(B)$, and hence the three sublcasses of well-bounded operators, uniquely decomposable well-bounded operators, type $(A)$ and type $(B)$, coincide with the class of well-bounded operators on this space. One of the most important questions in the theory is whether the spaces with this property are necessarily reflexive. Furthermore, it would be interesting to identify Banach spaces on which two subclasses of well-bounded operators coincide. We present some significant results towards the solution of these open problems. Some of the results also appear in [3].

Ricker [6] and [7] initiated the study of scalar-type spectral operators and wellbounded operators of type $(B)$ on GDP-spaces. He proved that every well-bounded operator of type $(B)$ on a GDP-space has a finite spectrum. We shall show that the Banach spaces on which every well-bounded operator operator of tyep $(B)$ has a finite spectrum are those which do not contain any complemented subspace with Schauder decomposition. Furthermore, we show that a Banach space contains no complemented subspaces with finite Schauder decompsoition if and only if every compact well-bounded operator on it has a finite spectrum. Pisier [5] constructed an example of such a Banach 
space. We also discuss well-bounded operators on hereditarily in-decomposable Banach spaces. Ricker [8, Theorems 1-5] gave a complete description of the structure and spectral properties of type $(B)$ well-bounded operators on such spaces. We shall show that the main results of [8] hold not only for well-bounded operators of type $(B)$, but also for general well-bounded operators.

\section{REFERENCES}

[1] H. Benzinger, E. Berkson and T.A. Gillespie, 'Spectral families of projections, semigroups, and differential oeprators', Trans. Amer. Math. Soc. 275 (1983), 431-475.

[2] E. Berkson and H.R. Dowson, 'On uniquely decomposable well-bounded operators', Proc. London Math. Soc. 322 (1971), 339-358.

[3] Q. Cheng and I. Doust, 'Well-bounded operators on nonreflexive Banach spaces', Proc. Amer. Math. Soc. 126 (1996), 799-808.

[4] Q. Cheng and I. Doust, 'The dual theory of well-bounded operators', J. Operator Theory 37 (1997), 35-50.

[5] G. Pisier, 'Counterexample to a conjecture of Grothendieck', Act. Math. 151 (1983), 181-208.

[6] W. Ricker, 'Spectral operators of scalar-type in Grothendieck spaces with the Dunford-Pettis property', Bull. London Math. Soc. 17 (1985), 268-270.

[7] W. Ricker. 'Well-bounded operators of type $(B)$ in a class of Banach spaces', J. Austral. Math Soc. Ser. A 32 (1987), 399-408.

[8] W. Ricker. 'Well-bounded operators of type (B) in H.I. spaces', Acta. Sci. Math. (Szeged) 59 (1994), 475-488.

[9] J.R. Ringrose, 'On well-bounded operators II', Proc. London Math. Soc. 313 (1963), 613-638.

[10] J.K. Turner, 'On well-bounded and decomposable oeprators', Proc. London Math. Soc. 3 37 (1978), 521-544.

Department of Mathematics

Jingzhou Teacher's College

Jingzhou City, Hubei

China
Current address:

Department of Mathematics

Jingzhou Teacher's College

Jingzhou, Hubei

China 